\title{
Estudo Comparativo de Instrumentos de Avaliação de Affordances no Contexto das Interfaces Web
}

Comparative study of affordances evaluation tools in the context of web interfaces

JARDIM FILHO, Airton Jordani; Mestre em Design; UDESC/UFSC

airton.jordani@gmail.com

LAUZER, Marshal Becon; Mestre em Design; Universidade FEEVALE

marshal.lauzer@gmail.com

\section{Resumo}

O presente estudo parte da premissa que a interface de uma página web é uma ferramenta de mediação entre determinado software e o usuário. Para cumprir sua função, no entanto, é fundamental que a interface seja estruturada de forma a oferecer ao usuário condições para a realização desta tarefa. Entre os requisitos para que uma interface cumpra seu objetivo principal destaca-se a necessidade de indicar o "para-que-serve" de forma clara ao usuário. O nome deste atributo é "affordance". Este trabalho busca identificar instrumentos para avaliação de affordances e interfaces web. Os resultados obtidos nesta pesquisa apresentam três ferramentas para avaliação de affordances. Cada uma das ferramentas possui características próprias e o comparativo entre elas está esquematizado em forma de quadro, onde são apresentados o tipo de instrumento de avaliação, o que ele se propõe a avaliar e, ainda, considerações a respeito de cada uma destas ferramentas.

Palavras Chave: Affordance; Interface; Web; Instrumentos de Avaliação.

\begin{abstract}
This study assumes that a web page interface is a mediation tool between software and user. To fulfill its function, however, it is essential an interface structured allow user to do this task. The requirement for an interface to fulfill its main objective is to indicate the "what-for" clearly. The name of this attribute is "affordance". This paper aims to identify tools for evaluate affordances and web interfaces. The results of this research presents three tools to evaluate affordances. Every tool has its own characteristics and the comparison between them is outlined in table form, where are presented by the type of assessment tool, what it aims to evaluate and also considerations about each of these tools.
\end{abstract}

Keywords: Affordance; Interface; Web; Evaluation Tools. 


\section{Introdução}

Uma página web - e, mais especificamente, sua interface - são, por princípio, uma ferramenta de mediação, entre software e o usuário. É por meio dela que o usuário realiza tarefas com o uso do software. Acessar seu extrato bancário, fazer compras, procurar notícias sobre assunto de seu interesse, entre outros. Muitas são as tarefas possíveis de serem realizadas através da interface. Mas, para tanto, é fundamental que a interface web do software seja estruturada de forma a oferecer ao usuário condições para a realização da tarefa.

Por outro lado, sabe-se, ainda, que entre os atributos necessários para que uma interface cumpra seu objetivo principal - que é mediar a interação homem-computador - pode-se destacar como preponderante que ela indique o "para-que-serve" de forma clara e direta ao usuário. Em um primeiro contato, dar pistas ao usuário de como a interação deve ser iniciada deveria estar entre as principais preocupações dos desenvolvedores de interface, pois da impressão inicial depende toda a interação. A terminologia usada para nomear essa indicação do "para-que-serve", segundo Norman (2006), é "affordance". O conceito de affordance, aqui apresentado, de maneira resumida, pode ser entendido como a possibilidade de ação ou de uso que o usuário percebe ser capaz de fazer com determinado objeto.

De que forma o desempenho das affordances, enquanto facilitadoras da interação, pode ser mensurado? Que ferramentas estão disponíveis para realizar a medição? Qual ferramenta é a mais adequada para determinado caso? Estas são algumas das perguntas que este trabalho se propõe a tentar auxiliar os pesquisadores do campo do design de interação a responder. Neste texto serão apresentadas as conceituações de interface, interface gráfica do usuário e affordances - bem como sua classificação e alguns instrumentos de avaliação desenvolvidos para tal.

\section{Interface}

Interface é um termo que está presente no dia-a-dia de grande parte das pessoas. Em um conceito amplo, interface é uma superfície que delimita dois corpos, espaços ou fases. Também pode ser vista como um dispositivo, físico ou lógico, responsável pela adaptação entre dois sistemas independentes. Embora o senso comum relacione a presença da interface a dispositivos eletrônicos - sejam eles smartphones, tablets, notebooks, entre outros - ela está presente em dispositivos de qualquer natureza, inclusive produtos não digitais. Segundo Bonsiepe (1997, p. 12), "a interface revela o caráter de ferramenta dos objetos e o conteúdo comunicativo das informações. A interface transforma objetos em produtos, [...] transforma sinais em informação interpretável."

Uma palavra-chave no que tange à interface é mediação. A interface permite, promove, regula e media processos que podem ser ou não relacionais atuando, quando necessário, como tradutor entre esses processos, como forma de permitir que ambos comuniquem-se entre si, através do entendimento e da habilidade em responder corretamente aquilo que o seu interlocutor pergunta e vice-versa (Grossmann, 2011).

Levy (1993, p. 181), por seu turno, afirma que "tudo aquilo que é tradução, transformação, passagem, é da ordem da interface", pois nem sempre as duas partes envolvidas nesse processo de comunicação se expressam através da mesma maneira. Daí a relação direta com a tradução, ou seja: para que a outra parte de um diálogo possa entender sua mensagem, muitas vezes é necessário que aquilo que foi dito em determinada linguagem seja traduzido para a da outra parte, pois esta pode não dominar a referida linguagem. 
É frequente a associação da palavra interface a computadores, como se antes da popularização da informática não houvessem interfaces. Steve Johnson, pioneiro teórico das interfaces digitais, no livro "Cultura da Interface" (2001, p. 17), ao tratar do assunto, questiona: "Mas, afinal, que é exatamente uma interface? Em seu sentido mais simples, a palavra se refere a softwares que dão forma à interação entre usuário e computador". Mesmo influenciado pelo senso comum sobre um conceito de interfaces ligado a computadores, Johnson já buscava um conceito mais abrangente pois, segundo o autor, a interface atua como uma espécie de tradutor, mediando entre duas partes, tornando uma sensível para outra.

Interface é um conceito do qual a ciência da computação apropriou-se e, posteriormente, o senso comum adotou como sendo oriundo do universo dos computadores.

No entanto, a interface gráfica teve sua origem na informática - também conhecida como "interface gráfica do usuário" (do inglês, Graphic User Interface ou, simplesmente, GUI). A GUI de um sistema computacional é composta por metáforas, imagens e conceitos de interação usados para expressar funcionamento e significado na tela do usuário. Ela também inclui as características visuais detalhadas de cada componente da interface gráfica e a sequência de interações ao longo do tempo que produzem a aparência e a atmosfera características das páginas web e das relações enlaçadas do hipertexto (Lynch \& Horton, 2004).

\section{Interface Gráfica do Usuário}

Apesar do conceito original de interface - de maneira simplificada - remeter a figura de um interlocutor entre diferentes linguagens, esse mesmo senso comum entende a GUI como um sinônimo de interface. Os antigos caracteres verdes sobre o fundo preto, em monitores de fósforo, formavam uma das primeiras interfaces dos computadores pessoais, chamadas de interface caractere, aquelas que popularizaram a informática levando o computador a figurar como mais um eletrodoméstico nos lares e como um equipamento nos escritórios - unindo funções que originalmente era realizadas com a ajuda da máquina de escrever, da agenda, do correio, entre outros.

No entanto, a palavra interface só começa a fazer parte, efetivamente, do vocabulário desse usuário contemporâneo de computadores, depois do surgimento da GUI, quase ao mesmo tempo em que o mouse se tornava um acessório obrigatório aos computadores pessoais. Isso permitiu que a própria GUI contasse com um sistema de interação diferente, baseado em apontar e clicar.

As interfaces computacionais ganharam notoriedade e popularidade quando elas deixaram de ser somente físicas, caracterizadas por botões, teclas e caracteres (estes últimos visíveis nos monitores de computadores), e assumiram o aspecto gráfico-visual, com janelas, ícones e menus. As interfaces gráficas foram as grandes responsáveis pela evolução e popularização dos sistemas computacionais, na medida em que possibilitaram o uso destes sistemas de modo mais intuitivo (Rocha, 2014, p. 58).

As GUls e sua capacidade de mimetizar o mundo real através de metáforas inspiradas no dia a dia do mundo real passaram a fazer parte não apenas da rotina dos escritórios: embora careça de conexão direta com a realidade doméstica dos usuários - pois pastas, mesa de trabalho, arquivos, documentos e outras metáforas utilizadas nas GUls pioneiras são objetos tipicamente encontrados no cotidiano de um escritório, e não da vida doméstica - ainda assim a interface gráfica surgiu como uma solução mais adequada à interlocução com a máquina do que caracteres verdes sobre fundo preto. 
Johnson (2001, p. 18) lembra que um computador é um sistema simbólico sob todos os aspectos. Aqueles pulsos de eletricidade são símbolos que representam zeros e uns, que por sua vez representam simples conjuntos de instrução matemática, que por sua vez representam palavras ou imagens, planilhas e mensagens de e-mail. O enorme poder do computador digital contemporâneo depende dessa capacidade de auto representação. Na maioria das vezes, essa representação assume a forma de uma metáfora. Uma sequência de zeros e uns é substituída pela metáfora de uma pasta virtual que reside num desktop virtual. Atualmente ainda existem sistemas operacionais para computadores pessoais que não utilizam uma GUI, no entanto a quantidade de usuários é de apenas $2 \%$ do mercado ${ }^{1}$. Este dado aponta a presença massiva e a importância da GUI nos computadores pessoais e, por consequência, nas interfaces web - que necessitam da GUI para serem exibidas ao usuário.

\section{Affordances}

No final dos anos 1970, o psicólogo norte-americano James Gibson publicou o livro "The ecological approach to visual perception". Nele, apresenta sua abordagem sobre a percepção visual, considerando como o ser humano se relaciona com o meio onde vive e porque as coisas se parecem como são. Gibson traz o conceito de affordances, "oportunidades para a ação" numa perspectiva de que não haveria representação ou manipulações simbólicas mentais envolvidas na cognição. $A$ percepção seria direta e toda a informação necessária para a ação oportuna dos organismos estaria disponível no ambiente, "uma abordagem ecológica e controversa para a época", conforme Sant'Anna (2013, p. 24).

As affordances são possibilidades de ação que o ambiente oferece ao agente. A formulação do conceito de affordance, possibilita o acesso a algumas novas ideias que auxiliam na compreensão da interação animal-ambiente, diferente de algumas vertentes tradicionais, causando repercussões e estimulando o debate científico e filosófico não só dos interessados nos estudos da percepção e informação, mas também da psicologia, sociologia, filosofia, ciência cognitiva, entre outras (Oliveira \& Rodrigues, 2006).

As propriedades são menos importantes do que os affordances. Ao perceber que a superfície é plana e sólida, o agente não percebe as qualidades, mas que a superfície é "caminhável", quando a cobra percebe a presença de sua presa, através da informação térmica, ela não detecta a temperatura em si, mas a direção do seu ataque; o indivíduo não percebe a cadeira e a caneta, mas a possibilidade de sentar e escrever, respectivamente (Oliveira \& Rodrigues, 2006). James Gibson (1977) afirma que o significado e a utilidade do ambiente são percebidos e estão diretamente ligados ao agente. Affordance é uma combinação específica das propriedades de suas substâncias e suas superfícies tomadas com referencia a um animal. No quadro a seguir, estão apresentados, de forma esquemática e sintética, os pressupostos básicos da teoria de Gibson para as affordances.

\footnotetext{
1 Segundo a NET Market Share, o Linux, sistema operacional para computadores pessoais capaz de ser rodado sem uma GUI detém uma faixa de mercado equivalente a 2,32\%. Fonte: http://www.netmarketshare.com. Acesso em: março/2018.
} 
Quadro 1 - Pressupostos da Teoria das Affordances de Gibson

\section{Os pressupostos da teoria de Gibson podem ser resumidos da seguinte forma:}

1. Affordances são percebidas diretamente, e não através da interpretação de dados sensoriais brutos inicialmente sem sentido

2. Affordances são propriedades relacionais; eles emergem na interação entre o animal e o meio ambiente

3. Affordances são independentes das necessidades situacionais do perceptor

4. Ambientes naturais e ambientes culturais não devem ser separados uns dos outros

5. A teoria das affordances está mais preocupada com a forma como as affordances são percebidas, do que com as affordances em si

Fonte: Elaborado pelos autores, com base em Kaptelinin \& Nardi (2012).

Embora Gibson tenha escrito sobre o assunto no final da década de 1970, este conceito ganhou projeção e destaque - principalmente entre designers - a partir da contribuição de Donald Norman, em seu livro "O design do dia-a-dia", lançado ainda nos anos 1980, mas traduzido para português apenas no meio dos anos 2000. Sant'anna (2013, p. 23) ao abordar o assunto, aponta que Norman, ao se apropriar de produção científica em diversos ramos das Ciências Cognitivas, construiu sua reputação como autor de referência no Design. Além dos últimos trabalhos sobre complexidade, o futuro e aspectos emocionais do Design, Norman é tido como o divulgador das affordances como teoria essencial para a área.

Ainda, segundo Sant'anna (2013), Donald Norman não apenas resgatou a teoria de Gibson, mas tratou de modificá-la para que esta pudesse responder de forma mais eficiente à realidade do projeto de produto. Norman apresentou, então, o conceito das affordances percebidas, que difere das affordances de Gibson. Ele desviou-se do conceito original de affordances, afirmando que elas são algo que é percebido pelo usuário, dependem da nossa intepretação mental dos objetos e, consequentemente, do conhecimento e experiências anteriores de cada indivíduo. As affordances percebidas indicariam o "para-que-serve" de um objeto e permitiriam compreendermos suas possibilidades de uso.

O quadro dois a seguir, mostra uma comparação entre o conceito de affordance descrito originalmente por Gibson e a atualização deste conceito apresentada por Norman. É importante observar que, enquanto Gibson afirma que o ator não necessita de experiência ou conhecimento para perceber a affordance, a versão de Norman diz que pode sim, depender da experiência, conhecimento ou cultura do ator. 
Quadro 2 - Comparação das affordances conforme definido por Gibson e por Norman

\begin{tabular}{ll}
\multicolumn{1}{c}{ Affordances de Gibson } & \multicolumn{1}{c}{ Affordances de Norman } \\
\hline - Ofertas ou possibilidades de ação no ambiente em & - Propriedades percebidas que podem ou não \\
relação às capacidades de ação de um ator & existir realmente \\
- Independente da experiência, cultura ou conhecimento & - Sugestões ou dicas de como usar as propriedades \\
do ator, ou sua habilidade de perceber & - Pode ser dependente da experiência, \\
- Existência binária - uma affordance existe ou não existe & conhecimento ou cultura do ator \\
& Pode tornar uma ação difícil ou fácil \\
\hline
\end{tabular}

Fonte: Elaborado pelo autor, com base em McGrenere \& Ho (2000).

Norman (2006, p. 13), no prefácio do livro "O design do dia-a-dia", que as affordances são as pistas deixadas pelo designer para que o usuário consiga intuir o que deve ser feito naquela interface para executar a ação desejada. Weinschenk (2011, p. 15) usa, como exemplo para explicar o conceito, uma maçaneta de porta que não funciona adequadamente: a alça parece dizer que você deve puxar, mas na verdade você precisa empurrar. No mundo real os objetos se comunicam conosco e dão suas pistas de como devemos interagir com eles. Se o objeto dá pistas que não funcionam, o usuário pode se irritar e frustrar. Se o designer deseja que as pessoas ajam de determinada maneira ao lidar com determinado objeto - não importando se estamos falando em objetos reais ou virtuais - é necessário se certificar que o usuário possa facilmente perceber, descobrir e interpretar o que o objeto é e o que pode e deve fazer com ele. Conforme descreve o próprio NORMAN,

as affordances podem sinalizar como o objeto deve ser movido, o que ele pode sustentar e se alguma coisa se encaixará em suas fendas, acima ou abaixo delas. Onde seguramos o objeto, que peças movemos e que peças são fixas? Affordances sugerem a variedade de possibilidades, as coerções limitam o número de alternativas. O uso atento da combinação de affordances e de restrições no design permite ao usuário determinar prontamente a sequência de ações a executar, mesmo numa situação nova (Norman, 2006, p. 112).

Nos anos 2000, absorvido pelos autores que abordavam o design de interação, o termo ganhou popularidade e aplicação na área de desenvolvimento de interfaces web. Embora refira-se a interface de maneira geral, o conceito impactou no desenvolvimento e, principalmente, na avaliação de interfaces web.

Preece, Rogers \& Sharp (2013), ao tratarem do design de interação listam o seus princípios do design. Os princípios são requisitos que deveriam ser levados em conta quando se projeta qualquer interface. As autoras abordam, ainda, alguns dos princípios elencados por Norman para o design e a usabilidade. Em destaque, dentre eles, está o conceito de affordance, juntamente com o feedback e as restrições - como sendo pontos críticos a serem considerados.

Aplicando-se às interfaces web, a partir do que descreve Norman (2006), é possível afirmar que as affordances estão diretamente ligadas à satisfação do usuário. Se sua presença serve como pista do que deve ser feito, sua falta pode gerar - durante a interação entre usuário e interface - um sentimento de frustração que, por vezes, pode impactar negativamente na satisfação do usuário com determinado artefato. 
Cabe aqui um adendo: em artigo publicado no ACM Interactions em 2008 e disponibilizado em seu site pessoal ${ }^{2}$, Norman tenta se desvencilhar do termo affordance, sugerindo que outro conceito - denominado "signifiers"("significante" em tradução nossa) - devesse ser usado, por ter maior abrangência e estar vinculado não apenas ao objeto ou interface, mas diria respeito ao ambiente e suas relações "sociais" dos agentes com o meio. O autor se mostra um tanto incomodado com a abrangência que suas ideias tomaram, fazendo um apelo para que os desenvolvedores passassem a usar este novo termo ao invés do anterior (NORMAN, 2008). Cabe dizer, a título de curiosidade, que em seu livro de 2004, intitulado "Design Emocional" ele já fazia uma espécie de mea culpa, questionando os termos apresentados em outro livro seu - Design do dia-a-dia - e o uso exacerbado que os designers estavam fazendo deles.

\section{Classificação das Affordances}

Em artigo intitulado "Technology affordances", William W. Gaver trata do conceito de Norman, aplicado às interfaces. Segundo Gaver (2002, p. 79), affordances são propriedades do mundo que são compatíveis e relevantes para as interações das pessoas. De maneira geral, o autor as classifica em três tipos: percebida, escondida e falsa. Quando são perceptíveis, elas oferecem uma ligação direta entre percepção e ação. Aquilo que se percebe tem relação direta com aquilo que resulta da ação. Já as escondidas e falsas podem levar o usuário a cometer erros durante a realização de sua tarefa.

Figura 1 - Separar affordances da informação disponível sobre elas permite a distinção entre rejeição correta e affordances percebidas, escondidas e falsas.

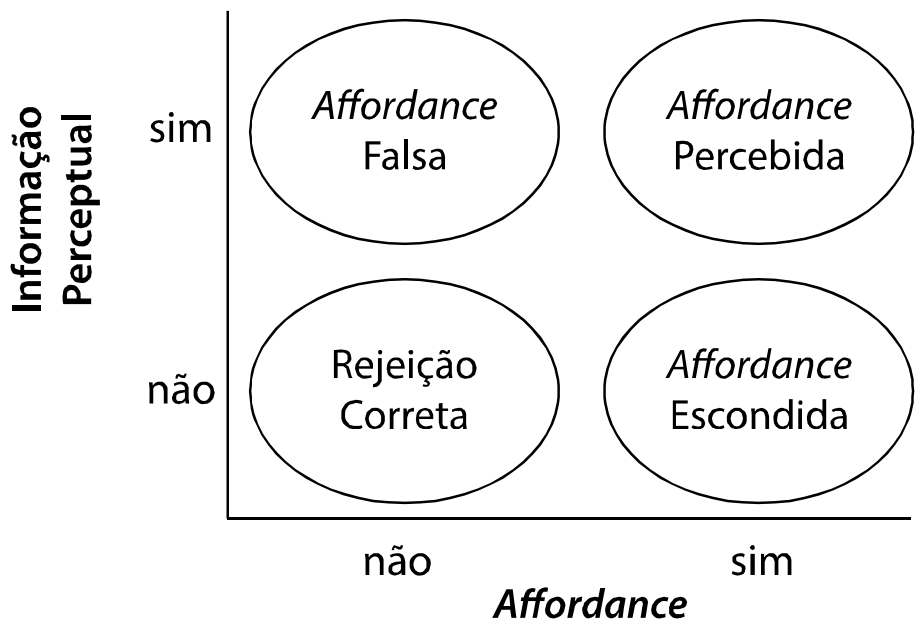

Fonte: Elaborado pelos autores, adaptado de Gaver (2002, p. 80)

Gaver (2002, p. 82) traz, ainda, o conceito de affordances sequenciais: situações em que uma affordance perceptível leva a novas informações contendo affordances, encadeadas. Este conceito, embora exemplificado pelo autor com as barras de rolagem de sistemas operacionais de computador, pode ser diretamente aplicado à interfaces web. A partir do conceito de hipertexto -

\footnotetext{
${ }^{2}$ Disponível em: https://www.jnd.org/dn.mss/signifiers_not_affordances.html. Acesso em 21/03/2018.
} 
ao qual toda a interface web está fortemente vinculada - o encadeamento de affordances pode tornar a navegação web ainda mais bem sucedida, do ponto de vista do usuário que realiza a tarefa.

Se as affordances descritas por Norman (2006) e classificadas por Gaver (2002) forem levadas em consideração como uma prioridade no processo de desenvolvimento de uma interface $w e b$, é possível concluir que o uso das affordances em sua plenitude estará diretamente relacionado a uma melhoria da experiência do usuário.

\section{Instrumentos de Avaliação das Affordances}

Em artigo intitulado "Technology affordances", William W. Gaver trata do conceito de Norman, aplicado às interfaces. Segundo Gaver (2002, p. 79), affordances são propriedades do mundo que são compatíveis e relevantes para as interações das pessoas. De maneira geral, o autor as classifica em três tipos: percebida, escondida e falsa. Quando são perceptíveis, elas oferecem uma ligação direta entre percepção e ação. Aquilo que se percebe tem relação direta com aquilo que resulta da ação. Já as escondidas e falsas podem levar o usuário a cometer erros durante a realização de sua tarefa.

\subsection{A IXD Checklist como ferramenta de avaliação de affordances em interfaces digitais, por Legaspi \& Jakhu (2018)}

Como resultado de uma revisão bibliográfica que buscou ferramentas para avaliação de affordances em interfaces digitais, foi encontrada uma análise de protótipo de livro digital, realizada por Dick \& Gonçalves (2014). Os autores do estudo utilizaram como ferramenta uma parte do IXD Checklist, de Legaspi \& Jakhu (2016). O IXD Checklist é uma ferramenta para avaliação, baseada em parâmetros reconhecidos pelo design de interação. Esses parâmetros dividem a ferramenta em partes, cada uma delas responsável por avaliar um aspecto diferente da interface. São eles: affordance, feedback, simplicidade (simplicity), estrutura (structure), consistência (consistency), tolerância (tolerance) e acessibilidade (acessibility).

Uma das funcionalidades da ferramenta permite filtrar aquilo que se deseja avaliar na interação, reduzindo e tornando a lista de parâmetros específica para determinado foco da interação. Os cinco filtros disponíveis são todos (all), interface, iconografia (iconography), tipografia (typography), interação (interaction) e navegação (navigation). Ao selecionar apenas 'interface', a ferramenta restringe os parâmetros à affordance, feedback, consistência, tolerância e acessibilidade. Por tratar-se de estudo recente, ainda não foram encontrados registros sistematizados de seu uso, tampouco há em seu site um maior detalhamento da utilização da ferramenta. No entanto, a julgar pelos parâmetros propostos, pode oferecer uma significativa contribuição na avaliação de affordances em interfaces digitais.

\subsection{A força de uma affordance, por Rosling (2011)}

Hans Rosling defende, no capítulo 3, do livro "Visual Interface Design for Digital Cultural Heritage" (2011) que a melhor maneira de avaliar as affordances é a partir de sua força em determinado contexto de uso por um grupo particular de usuários. A partir desta abordagem, o autor sugere que os pesquisadores possam começar a comparar a força da affordance em diferentes tipos de ferramentas de software, fornecendo informações essenciais aos designers de interface, nos diferentes estágios durante o processo de desenvolvimento. Ainda segundo Rosling (2011, p. 75), "dada a necessidade de especificar os fatores relacionais significativos que caracterizam a força 
de uma affordance, é possível distinguir cinco fatores que, juntos, representam os aspectos relacionais do objeto, do observador e da dinâmica do contexto:

a) capacidade tácita - o quão bem este recurso funciona?;

b) disponibilidade (inclui potencial situado, conscientização e apoio do ambiente) - o quão fácil você diria que é acessar este recurso? o quão bem este recurso é suportado no contexto em que você normalmente o utilizaria?;

c) tendência (inclui motivação, preferencia e hábito) - o quão ciente você estava deste recurso e de seu uso? se você teve escolha entre esse e outros recursos que fornecem a mesma função, como você avaliaria sua preferência pessoal por este recurso? o quão motivado você se sente a utilizar este recurso?;

d) habilidade - como você classificaria a si mesmo como usuário deste recurso?;

e) suporte institucional - se você usou este protótipo em seu trabalho, você conhece pessoas que poderiam the auxiliar no caso de você precisar de ajuda?.

Esses fatores em conjunto podem ser usados como base para questões de estudo de usuário que ajudam o designer para compreender os aspectos relacionais da força da affordance de forma operacional. Uma propriedade relevante e útil compartilhada por todos esses fatores é que se qualquer um deles é negado, toda a affordance desaparece (Rosling, 2011, p. 76). Diferente da IXD Checklist, a proposta de Rosling é avaliar a affordance. Isso faz desta proposta uma ferramenta mais específica, com parâmetros mais detalhados para a avaliação.

\subsection{Diagnosticando affordances, por Conn (1995)}

Apesar de ser um estudo com mais tempo de existência, o artigo de Alex Paul Conn apresenta um vasto estudo a respeito das affordances e seu comportamento em uma interface de aplicativo. $\mathrm{O}$ autor propõe um modelo baseado em mini-modos ${ }^{3}$. Esses mini-modos definem os estados nos quais as operações indicadas pelas affordances se tornam disponíveis, mas os minimodos não são "donos" das funções. Uma função em particular pode estar disponível em mais de um mini-modo. $O$ indicador de affordance pode ser um elemento especial ou ele pode ser um grupo de ícones relacionados. Ainda segundo Conn (1995), cada affordance consistirá de alguns ou todos os seguintes componentes:

a) Existência - indica a existência de uma função, operação ou estado em potencial;

b) Propósito - Indica (normalmente de forma visual) tanto o propósito quanto o uso de uma função, operação ou estado;

c) Disponibilidade - Indica se o usuário pode ativar a função, operação ou estado;

d) Ativação - Indica que o sistema reconhece ou aceita a seleção feita pelo usuário;

e) Aceitação - Demonstra o estado atual de um atributo ou mini-modo;

f) Padrão (default) - Demonstra as propriedades padrão atuais de um atributo ou estado;

g) Garantia - Assegura ao usuário que a affordance diz é realmente verdade;

h) Diagnóstico - Fornece informações adicionais sobre a disponibilidade ou estado de uma affordance.

\footnotetext{
${ }^{3}$ Uma determinada função é geralmente indisponível porque o programa de computador não está em um estado onde essa função faria sentido. Onde dois ou mais estados estão relacionados porque as suas operações ou funções são naturalmente amarradas juntas, nós definimos o conjunto de estados como um "mini-modo" (Conn, 1995, p. 2).
} 
Em seu trabalho, Alex Paul Conn destaca a importância de que os "style guides" devem ser desenvolvidos sempre com as affordances em mente. Por fim, em seu trabalho, Conn (1995) sugere algumas heurísticas - ou recomendações - a serem seguidas quando do projeto de uma interface:

a) Disponibilizar affordances;

b) Usar consistentemente os mini-modos;

c) Permitir aos usuários a customização de barras de tarefas para agrupar affordances e para criar novas affordances;

d) Indicar quando um estado está ativo ou ligado;

e) Usar consistentemente os paradigmas de affordances no mesmo aplicativo;

f) Usar affordances de forma cooperativada ou agrupada para ajudar a reduzir ambiguidades.

Ainda que se trate de um estudo com mais de 20 anos, o trabalho de CONN traz importantes sugestões a respeito da relação entre interface e affordances, sugerindo tanto como avaliá-las, quanto indicando heurísticas de boas práticas. É importante ressaltar que, à época do estudo, Alex Paul Conn era pesquisador da Digital/Compaq Computer Corporation. No meio dos anos 1990, a Compaq era uma verdadeira potência mundial em computadores e uma das líderes em inovação e pesquisa. Antes da era Google, o maior buscador de internet do mercado era o Alta Vista, de propriedade da Compaq. Essa informação contextualiza e demonstra o grande interesse em pesquisa e desenvolvimento que a empresa tinha naquela época. Este estudo, por si só, já seria um excelente ponto de partida para a pesquisa com relação à interfaces e affordances.

\subsection{Quadro comparativo instrumentos de avaliação}

O quadro 3, a seguir, apresenta uma comparação dos instrumentos elencados neste trabalho de pesquisa. Através desta síntese, é possível comparar os diferentes perfis de cada uma das propostas. Enquanto Legaspi \& Jakhu (2016) disponibilizam sua ferramenta pela internet, com uma interface customizável, a proposta de Conn (1995) mesmo com mais de vinte anos, mas ainda assim, permanece relevante e possui a abordagem mais completa entre as ferramentas estudadas. Há, ainda, a ferramenta proposta por Rosling (2011) que, assim como Conn (1995), apresenta suas próprias heurísticas para avaliação das affordances. 
Quadro 3 - Comparativo Instrumentos De Avaliação

\begin{tabular}{ll}
\hline $\begin{array}{l}\text { Ferramenta / } \\
\text { Autor(es) }\end{array}$ & $\begin{array}{l}\text { Tipo de instrumento de } \\
\text { avaliação }\end{array}$ \\
\hline $\begin{array}{l}\text { IXD Checklist, } \\
\text { por Legaspi \& }\end{array}$ & $\begin{array}{l}\text { Parâmetros reconhecidos } \\
\text { pelo design de interação } \\
\text { Jakfordance, feedback, }\end{array}$ \\
& $\begin{array}{l}\text { simplicidade, estrutura, } \\
\text { consistência, tolerância e } \\
\text { acessibilidade. }\end{array}$
\end{tabular}

O que se propõe a avaliar

Diferentes aspectos de

uma interface, podendo

ser utilizada

modularmente. No caso

deste estudo, adotou-se,

assim como em Dick \&

Gonçalves (2014), se

recomenda o uso do

parâmetro affordance.

\section{Considerações sobre a \\ ferramenta}

\author{
Por tratar-se de estudo recente, \\ ainda não existem registros \\ sistematizados de seu uso, \\ tampouco há em seu site um \\ maior detalhamento da \\ utilização. No entanto, pode \\ oferecer uma significativa \\ contribuição na avaliação de \\ affordances em interfaces \\ digitais.
}

Affordance
Strenght,
por Rosling (2011)

\section{Cinco fatores que} representam os aspectos relacionais do objeto, do observador e da dinâmica do contexto: a) capacidade tácita; b) disponibilidade (inclui potencial situado, conscientização e apoio do ambiente); c) tendência (inclui motivação, preferencia e hábito); d) habilidade; e) suporte institucional.

\section{Comparação da força da} affordance em diferentes tipos de ferramentas de software, fornecendo informações essenciais aos designers de interface, nos diferentes estágios durante o processo de desenvolvimento

\section{Esses fatores em conjunto podem ser usados como base para questões de estudo de usuário que ajudam o designer para compreender os aspectos relacionais da força da affordance de forma operacional. Diferente da IXD Checklist, a proposta de Rosling é avaliar a affordance. Isso faz desta proposta uma ferramenta mais específica, com parâmetros mais detalhados para a avaliação.}
Uma proposta abrangente: propõe uma forma de avaliar e apresenta recomendações de uso. Na época do estudo, Alex Paul Conn era pesquisador da Digital/Compaq. No meio dos anos 1990, a Compaq era uma das líderes em inovação e pesquisa. Antes da era Google, o maior buscador de internet do mercado era o Alta Vista, de propriedade da Compaq. Essa informação contextualiza o interesse em pesquisa e desenvolvimento que a empresa tinha naquela época.

agrupada para reduzir ambiguidades.

Heurísticas: a)
Disponibilizar affordances;
b) Usar consistentemente
os mini-modos; c) Permitir
a customização de barras
de tarefas para agrupar e
criar novas affordances; d)
Indicar quando um estado
está ativo ou ligado; e)
Usar consistentemente os
paradigmas de
affordances no mesmo
aplicativo; f) Usar
affordances de forma
cooperativada ou
agrupada para reduzir
ambiguidades.

\section{Modelo de affordanc} disponíveis.

Apesar de ter 20 anos, 0 trabalho de Conn traz importantes sugestões de boas práticas

Fonte: Elaborado pelos autores, adaptado de Legaspi \& Jakhu (2018), Rosling (2011) e Conn (1995). 


\section{Considerações Finais}

No presente trabalho, em um primeiro momento, buscou-se a conceituação de termos que, embora presentes no dia-a-dia de muitas pessoas pessoas, por vezes têm, no senso comum, um significado que não condiz com aquele utilizado no jargão técnico, tanto do design, quanto de outras áreas relacionadas ao design de interação. Foi o caso dos conceitos de interface e interface gráfica do usuário (GUI).

A seguir, buscou-se traçar um histórico do termo affordance, sua origem e sua posterior redescoberta, enquanto facilitadora da interação. Foi feito, ainda, um comparativo do uso do termo em seus diferentes contextos históricos, no final dos anos 1970, com James Gibson e, posteriormente, a partir do meio dos anos 1980, com Donald Norman.

A partir da base teórica levantada a respeito das affordances, fez-se uma revisão bibliográfica em busca de maneiras de mensurar sua força e pertinência. Buscou-se, ainda, ferramentas que pudessem ser utilizadas nessa medição. Foram encontrados os estudos de Legaspi \& Jakhu (2016), Rosling (2011) e Conn (1995).

Uma síntese destes estudos - tendo como critérios o tipo de instrumento de avaliação e o que a ferramenta se propõe a avaliar, somadas às considerações sobre cada ferramenta - gerou o Quadro 3, apresentado no ítem 6.4 deste trabalho de pesquisa. Em resumo, o quadro mostra que cada uma delas tem suas peculiaridades e aplicações possíveis, dependendo do que se deseja avaliar. Os possíveis desdobramentos desta pesquisa incluem a continuidade da busca por ferramentas para avaliação de affordances, bem como a execução de testes para validação destas ferramentas.

\section{Referências}

BONSIEPE, G. Do material ao digital. Florianópolis: LBDI, 1997.

. Design, cultura e sociedade. São Paulo: Blucher, 2011.

CAUDURO, F. V. O Digital na Comunicação. In: LEVACOV, M. et al. Tendências na Comunicação. Porto Alegre: L\&PM, 1998.

CONN, A. P. Diagnostic affordances: heuristics for Enhancing Interface Information. In: CHI '95 Proceedings of the SIGCHI Conference on Human Factors in Computing Systems. Pages 186-193, 1995.

DICK, Mauricio Elias; GONÇALVES, Berenice Santos; "A análise de livro digital: uma visão de suas affordances ", p. 3275-3286 . In: Anais do 11을 Congresso Brasileiro de Pesquisa e Desenvolvimento em Design [= Blucher Design Proceedings, v. 1, n. 4]. São Paulo: Blucher, 2014.

GAVER, William W. Technology affordances. Cambridge, 2002. Disponível em: < https://www.Iri.fr/ mbl/Stanford/CS477/papers/Gaver-CHI1991.pdf >. Acesso em: 17 mar. 2018

GIBSON, J. J. The theory of affordance. In: SHAW, R.; BRANSFORD, J. Perceiving, acting and knowing: toward an Ecological psychology. New Jersey: Laurence Erlbaum Associates, p. 67-82, 1977.

. The Ecological Approach to Visual Perception. Boston: Houghton Mifflin, 1979.

GROSSMANN, M. Museu como interface. In: GROSSMANN, M. \& MARIOTTI, G. Museum Art Today / Museu Arte Hoje, São Paulo, Hedra \& Forum Permanente, 2011, p. 193-221. 
JOHNSON, S. Cultura da interface: como o computador transforma nossa maneira de criar e comunicar. Rio de Janeiro: Jorge Zahar Ed. 2001.

KAPTELININ, V.; NARDI, B. Affordances in HCl: Toward a Mediated Action Perspective. In: CHI 2012, May 5-10, Austin, Texas, USA. P. 967-976, 2012.

LEGASPI, A.; JAKHU, A. IxD Checklist. Disponível em: < http://ixdchecklist.com/ >. Acesso em: 17 mar. 2018.

LÉVY, Pierre. As tecnologias da inteligência - o futuro do pensamento na era da informática. Rio de Janeiro: Ed. 34, 1993.

LYNCH, P. J.; HORTON, S. Guia de estilo da web: princípios básicos de design para criação de websites. Barcelona: Editorial Gustavo Gili, 2004.

MCGRENERE, J.; HO, W. Affordances: Clarifying and Evolving a Concept. In: Proceedings of Graphic Interface 2000, Montreal, May-2000, p. 1-8.

NORMAN, D. O design do dia-a-dia. São Paulo: Rocco. 2006.

. Design emocional. São Paulo: Rocco. 2008.

NORMAN, D. THE WAY I SEE IT: Signifiers, Not Affordances. Interactions, v. 15, n. 6, p. 18-19, nov. 2008.

OLIVEIRA, F. I. S.; RODRIGUES, S.T. Affordances: a relação entre agente e ambiente. In: Ciências \& Cognição. 2006. Vol. 09, p. 120-130.

PREECE, J.; ROGERS, Y.; SHARP, H. Design de interação: além da interação homem-computador. Porto Alegre, RS: Bookman, $3^{\text {a }}$ Ed, 2013.

ROCHA, C. Pontes, janelas e peles: cultura, poéticas e perspectivas das interfaces computacionais. Goiania: UFG, 2014.

ROSLING, H. Is This Thing Working? The Study of New Affordances. In: RUECKER, S.; RADZIKOWSKA, M.; SINCLAIR, S. (Orgs.) Visual Interface Design for digital Cultural Heritage: A Guide to RichProspect Browsing. Ashgate: MPG Books Group/UK, 2011.

SANT'ANNA, H. C. Design sem designer. Serra: Edição do autor, 2013.

WEINSCHENK, S. 100 Things Every Designer Needs to Know About People. Berkeley: New Riders, 2011. 